\title{
Abnormalities of cortical-limbic-cerebellar white matter networks may contribute to treatment-resistant depression: a diffusion tensor imaging study
}

Hong-jun Peng ${ }^{1,2+}$, Hui-rong Zheng ${ }^{3+}$, Yu-ping Ning ${ }^{2+}$, Yan Zhang ${ }^{1}$, Bao-ci Shan ${ }^{4}$, Li Zhang ${ }^{1}$, Hai-chen Yang ${ }^{1}$, Jun Liư ${ }^{5}$, Ze-xuan Li', Jian-song Zhou', Zhi-jun Zhang ${ }^{6}$ and Ling-jiang Li ${ }^{1,7^{*}}$

\begin{abstract}
Background: White matter abnormalities can cause network dysfunction that underlies major depressive disorder (MDD). Diffusion tensor imaging (DTI) is used to examine the neural connectivity and integrity of the white matter. Previous studies have implicated frontolimbic neural networks in the pathophysiology of MDD. Approximately 30\% of MDD patients demonstrate treatment-resistant depression (TRD). However, the neurobiology of TRD remains unclear.

Methods: We used a voxel-based analysis method to analyze DTI data in young patients with TRD $(n=30$; 19 males, 11 females) compared with right-handed, age- and sex-matched healthy volunteers $(n=25 ; 14$ males, 11 females).

Results: We found a significant decrease in fractional anisotropy (FA) (corrected, cluster size $>50$ ) in the left middle frontal gyrus (peak coordinates [-18 46-14]), left limbic lobe uncus (peak coordinates [-18 2-22]), and right cerebellum posterior lobe (peak coordinates [26-34-40]). There was no increase in FA in any brain region in patients. We also found a significant negative correlation between mean regional FA values in the three areas and Beck Depression Inventory symptom scores.

Conclusions: We found significant differences in white matter FA in the frontal lobe, limbic lobe and cerebellum between TRD patients and controls. These data suggest that abnormalities of cortical-limbic-cerebellar white matter networks may contribute to TRD in young patients.
\end{abstract}

Keywords: Treatment-resistant depression, Diffusion tensor imaging, Fractional anisotropy, Voxel-based analysis method

\section{Background}

Major depression is a common condition and a leading cause of disability worldwide [1]. Approximately $5 \%$ of American adults are affected by depression each year, $30 \%$ of whom fail to respond to two or more types of antidepressant, a phenomenon termed treatment-resistant depression (TRD) [2-7]. The pathogenesis of major

\footnotetext{
* Correspondence: Ilj2920@163.com

${ }^{\dagger}$ Equal contributors

'Mental Health Institute, The 2nd Xiangya Hospital, Central South University, No. 139 Renmin Zhong Road, Changsha 410011, China

${ }^{7}$ Chinese University of Hong Kong, Hong Kong, China

Full list of author information is available at the end of the article
}

depressive disorder (MDD) and the pathogenic mechanism of TRD remain unclear. Techniques such as magnetic resonance imaging (MRI), especially diffusion tensor imaging (DTI), have revealed white matter abnormalities in multiple psychiatric disorders [8-10]. The white matter forms the basis of anatomical connectivity, and disruption of this connectivity can result in brain dysfunction underlying various psychiatric disorders $[11,12]$. DTI is a useful tool for examining and quantifying white matter microstructure, including the orientation and integrity of white matter tracts, by detecting the diffusion of water in neural tissue in vivo [10]. A high fractional anisotropy (FA)

\section{() Biomed Central}


reflects intact axonal membranes, myelin sheaths, and a parallel arrangement of neurofibrils. By contrast, a low FA reflects damaged integrity of the white matter [9].

Previous studies using DTI have mainly focused on affective disorders including MDD, BD [13,14], and young and geriatric depression $[15,16]$, and the results showed the abnormal brain regions include the superior, middle, and medial frontal gyrus $[9,16,17]$, the subgenual anterior cingulate cortex (ACC), amygdala [14], hippocampus [18], and basal ganglia [19]. These abnormal brain regions are predominantly located in the limbiccortical-striatal-pallidal-thalamic tract (LCSPT) [20,21], which is considered related to emotional behavior on the basis of its anatomical connectivity with visceral control structures that mediate emotional expression [19].

It remains unclear whether the pathogenesis of TRD is similar to various affective disorders, although there is some limited DTI evidence that abnormal brain areas in TRD include the LCSPT circuits, similar to general affective disorders [22]. In the present study, we used an explorative voxel-based analysis (VBA) method to investigate the white matter integrity of TRD patients in order to determine the specific microstructure alterations in TRD. We hypothesized that the changes in white matter FA in TRD are similar to general affective disorders involving abnormalities of the cortical-limbic or cortical-subcortical circuits, as well as other important areas related to emotional regulation.

\section{Methods}

\section{Subjects}

Thirty patients (mean age, $26.87 \pm 5.28$ years; mean disease course, $4.68 \pm 3.37$ years) fulfilled both our diagnostic criteria for a major depressive episode (DSM-IV) and the TRD criteria. We defined treatment resistance as failure to respond to at least two different classes of antidepressant given for a period longer than 4 weeks at the maximum recommended dose [23]. The patients were recruited from the inpatient and outpatient units at the Institute of Mental Health at the Second Xiangya Hospital of Central South University, and the sex- and age- matched healthy controls were recruited in the local community. After each subject was fully informed of the study, written informed consent was obtained. The protocol was approved by the Central South University ethics committee and the studies were carried out in accordance with the Declaration of Helsinki. Two experienced psychiatrists performed patient diagnosis independently. We excluded patients with other psychiatric axis-I or axis-II disorders, neurological disorders, and other clinically relevant abnormalities in laboratory examinations. The patients with a counter indication of MRI were also excluded. The Beck Depression Inventory (BDI) [24] was used to assess clinical symptoms.

\section{Diffusion tensor imaging data acquisition}

The DTI scans were performed at the Magnetic Centre of Hunan Provincial People's Hospital. Subjects wore a standard birdcage head coil when they lay supine in a 3.0-Tesla head scanner (Allegra, Siemens Medical System). We used foam pads to minimize head motion, and used ear-plugs to diminish the sounds of the scanner. We collected high-resolution T1-weighted wholebrain 3-D MRI data with a magnetization-prepared rapid-acquisition gradient echo sequence (MP-RAGE) using the following parameters: 144 sagittal slices; thickness, $1.0 \mathrm{~mm}$; $256 \times 256$ matrix; field of view, $256 \times 256 \mathrm{~mm}$; TE, $3.7 \mathrm{~ms}$; and TR, $2000 \mathrm{~ms}$. We also collected a diffusion-weighted data set with an echo planar image sequence using the following parameters: 45 transversal slices; 30 gradient directions; thickness, $3.0 \mathrm{~mm}$; no gap; $192 \times 192$ matrix; field of view, $240 \times 240 \mathrm{~mm}$; TE, $93 \mathrm{~ms}$; TR, $6046 \mathrm{~ms}$; b1, 0; and b2, $1000 \mathrm{~s} / \mathrm{mm}^{2}$.

\section{Magnetic resonance imaging data analysis}

Diffusion tensor images were pre-processed using previously published methods[25-27]. The diffusion data set was pre-aligned to correct for head motion, and the effects of gradient coil eddy currents were corrected using software tools from the FMRIB software library (http:// www.fmrib.ox.ac.uk/fsl). The resulting FA images were transformed into Montreal Neurological Institute standard space using Statistical Parametric Mapping (SPM2; Wellcome Department of Cognitive Neurology, London, UK). For each subject, the $\mathrm{b}=0$ images were coregistered with the structural T1 image; the same coregistration parameters were applied to the FA maps (in the same space as the $b=0$ images). Each individuals' T1 image was then normalized to the SPM T1 template (in Montreal Neurological Institute standard space), and the same normalization parameters were then applied to the coregistered FA images. All images were resampled with a voxel size of $2 \times 2 \times 2 \mathrm{~mm}^{3}$. The normalized FA images were smoothed with an $8 \mathrm{~mm}$ full-width at half-maximum Gaussian kernel to decrease spatial noise, and a mean image (FA template) was created.

\section{Statistical analysis}

Two-sample t-tests were performed between 30 TRD patients and 25 healthy controls on diffusion tensor images of FA using SPM2 software. An initial threshold of 50 voxels or greater, surviving a false discovery rate (FDR) threshold of $P<0.05$, was set [28]. We retrieved white matter FA values from these identified clusters with home-developed software, as previously published [29]. Data were analyzed using SPSS17.0 software. A multiple-correlation analysis was performed to estimate the relationship between the average FA values and BDI 
scores, age, and duration of disease. A statistical threshold of $P<0.05$ (two-tailed) was used.

\section{Results}

Clinical and demographic characteristics of the subjects There were no significant age, gender, or marriage-state differences between patients and healthy control subjects $(P>0.05)$ (see Table 1).

\section{Diffusion tensor imaging of treatment-resistant depression patients}

Voxelwise analysis revealed reduced FA in three areas in the TRD group compared with control subjects $(P<0.001$, uncorrected, cluster size $>50)$. One area was located at the left limbic lobe uncus with peak coordinates [-18 2-22], the second area was located at the left middle frontal gyrus [-18 46-14], and the third area was located at the right cerebellum posterior lobe [26-34 -40]. The three areas survived an FDR threshold of $P<0.05$ at the cluster level or the voxel level (Figure 1 and Table 2). The left middle frontal gyrus survived FDR correction at the voxel level $(P=0.018)$, and the other two areas survived correction at the cluster level $(P=0.015$ and $P=0.025$, respectively). There were no other regions of reduced or increased FA of statistical significance in the TRD group compared with the control group. Our results showed significantly reduced FA values in the left middle frontal gyrus, left limbic lobe uncus, and right cerebellum posterior lobe in TRD subjects compared with controls $(P<0.001$; see Figure 2$)$.

\section{Correlation between depressive symptom scores and fractional anisotropy values in treatment-resistant depression patients}

Significant negative correlations were found between depression symptom scores (BDI) and reduced FA values in the left middle frontal gyrus, right limbic lobe uncus, and right cerebellum posterior lobe regions of interest (Figure 3); the correlation coefficients were $-0.379(P=0.039),-0.46$ $(P=0.009)$, and $-0.450 \quad(P=0.027)$, respectively. In addition, Pearson correlations found no correlations between FA values in regions of interest of TRD subjects, and age and disease duration.

\section{Effects of gender on reduced white matter fractional anisotropy values in the three regions}

There were no significant differences between males and females for reduced FA values in the left middle frontal gyrus $(P=0.588)$, left limbic lobe uncus $(P=0.636)$, and right cerebellum posterior lobe $(P=0.207$; see Table 3$)$.

\section{Discussion}

We found significant differences in white matter FA between TRD patients and healthy subjects in the left middle frontal gyrus, left limbic lobe uncus, and right cerebellum posterior lobe. These data suggest that abnormal cortical-limbic-cerebellar white matter circuits may underlie the pathogenesis of TRD, which is partly consistent with previous studies on affective disorders that implicate abnormalities of the cortical-limbic circuits.

White matter abnormalities in the middle frontal gyrus and limbic lobe have been reported in numerous studies using the VBA or TBSS methods, including MDD, BD, young and geriatric depression, and first-episode and recurrent depression [12,14-17,30-35]; in these studies, abnormal cortical-limbic or cortical-subcortical circuits related to emotional regulation were used to interpret the mechanisms of affective disorders. In the corticallimbic model proposed by Mayberg [36], the dorsal compartment includes both neocortical and midline limbic elements, and is thought to regulate attentional and cognitive symptoms of depression involving apathy and psychomotor retardation, while the ventral compartment, composed of the limbic, paralimbic cortical, subcortical, and brainstem regions, is proposed to mediate the vegetative and somatic aspects of depression. Depression is considered to be related to failure of the coordinated interactions of the dorsal and ventral compartment [37-39]. In our study, the left middle frontal gyrus and left limbic lobe uncus belong to the dorsal and ventral compartments, respectively, and dysfunction of these two compartments can account for the disturbances of emotional behavior. Modern brain imaging studies have supported a pronounced role of cortical-limbic topdown mechanisms in the regulation of mood and depression therapy, including the positive effect of cognitive behavioral therapy on depression $[40,41]$.

Table 1 Clinical and demographic characteristics of patients TRD and HC

\begin{tabular}{lllll}
\hline Variable & HC $(\mathbf{n}=\mathbf{2 5})$ & & TRD group $(\mathbf{n}=\mathbf{3 0})$ & PD \\
\hline & Mean & SD & Mean & 5.28 \\
Age(y) & 28.24 & 4.98 & 26.77 & 0.29 \\
Gender (male/female)(n) & $14 / 11$ & & $19 / 11$ & 0.80 \\
Marriage (single/married)(n) & $15 / 10$ & & $16 / 14$ & 0.92 \\
Course(y) & & & 4.68 & 3.37 \\
BDI & & & 20.47 & 4.45 \\
\hline
\end{tabular}

$\mathrm{P}>0.05$. $H C$, healthy control; $S D$, standard deviation; $T R D$, treatment-resistant depression. 

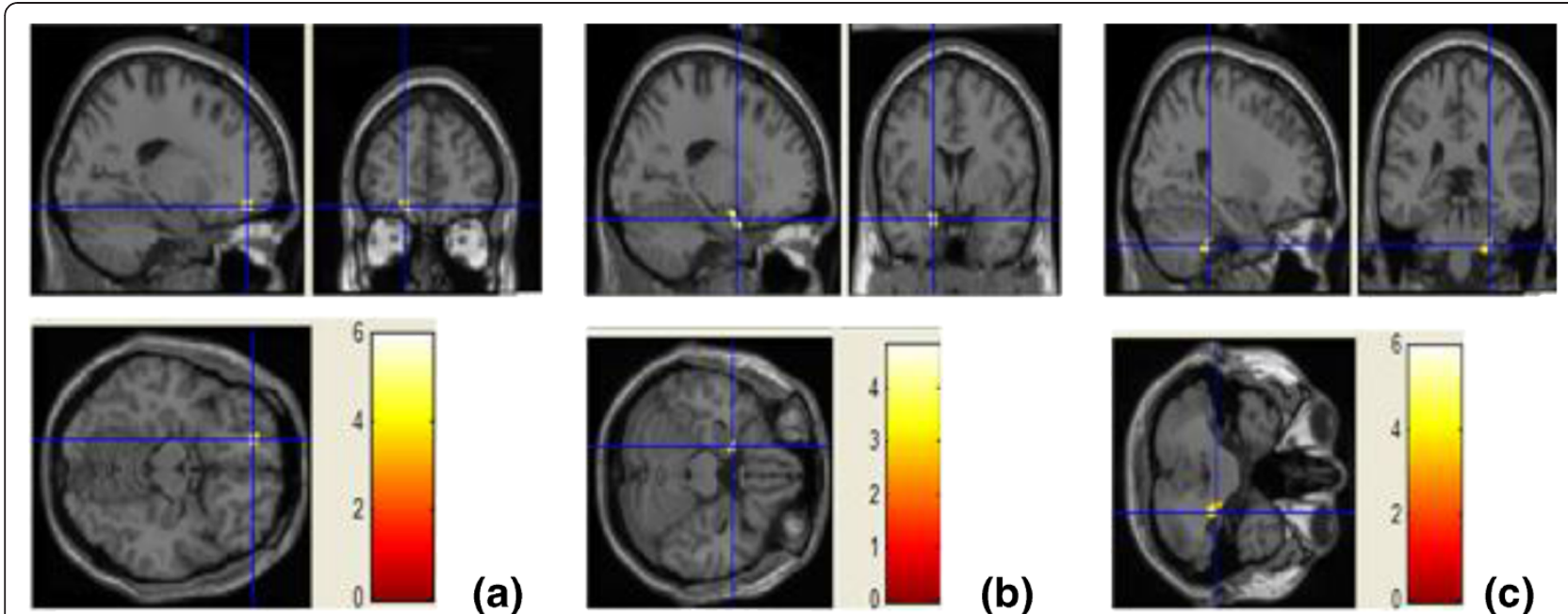

(a)

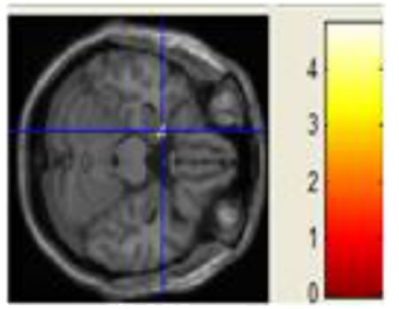

(b)
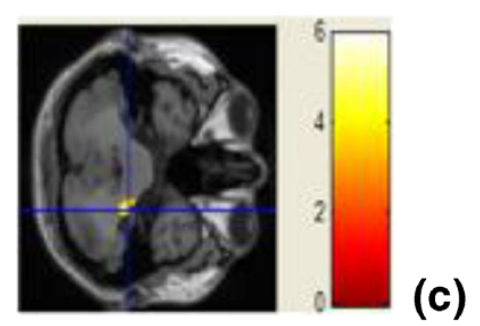

Figure 1 Areas of decreased fractional anisotropy extending over the left middle frontal gyrus (a), left limbic lobe uncus (b), and right cerebellum posterior lobe (c) in treatment-resistant depression patients compared with healthy control subjects.

The prefrontal cortex exerts a potent regulatory influence over the subcortical systems involved in the regulation of affective states $[42,43]$. Frontal-subcortical circuits such as the classic limbic-cortical-striatal-pallidal-thalamic (LCSPT) circuit, formed by connections between the orbital and medial prefrontal cortex, amygdala, hippocampal subiculum, ventromedial striatum, mediodorsal, and midline thalamic nuclei, and ventral pallidum, are considered to underlie emotional regulation $[20,21]$. These circuits can provide forebrain modulation over visceral control structures in the hypothalamus and brainstem, and their dysfunction can regulate the disturbances in autonomic regulation and neuroendocrine responses that are associated with mood disorders $[8,20,44]$. Our results showed abnormal white matter areas in the middle frontal gyrus and limbic lobe uncus, and the abnormal brain regions were located at or near LCSPT circuits. However, we did not find abnormalities in the classical brain areas such as the ACC, amygdala, or hippocampus often reported by previous studies of affective disorders [14,45], which may be related to our small sample size or rigorous threshold setting.

Besides the cortical-limbic or cortical-subcortical circuits, the cerebellum may also play an important role in emotional regulation. The traditionally held view is that the core functions of the cerebellum involve coordination, balance, and the motor component of speech regulation $[46,47]$. Recently, neuroanatomical studies have shown that the cerebellum is important for cognitive regulation through bidirectional pathways between the cerebellum and cortical structures, and cerebellar lesions can result in cerebellar-cognitive-affective syndrome, including executive, visual spatial, and linguistic impairments, and affective dysregulation. The cerebellum has extensive anatomic connections with many brainstem and forebrain structures. Several cerebellar-cerebral pathways are likely to be involved in emotional behavior, with several pathways emanating primarily from the cerebellar fastigial nuclei and terminating in various limbic structures including the hippocampus, amygdala, septal nuclei, mammillary bodies, and hypothalamus. Other potentially important pathways emanate from the ventrolateral dentate nucleus, travel to the thalamus (including dorsomedial nucleus), and terminate in the prefrontal cortex [48]. Doron et al. (2009) tracked connections between the cerebral peduncle and left hemispheric masks of the superior frontal gyrus, precentral gyrus, middle frontal gyrus, orbital frontal cortex, and two regions of the inferior frontal gyrus, supporting the relationship of the cerebellum with cognition and affection regulation [49]. In addition, the vermis of the cerebellum is recognized as an anatomical part of the limbic cerebellum, and vermis lesions often cause

Table 2 Brain regions with significantly lower fractional anisotropy

\begin{tabular}{|c|c|c|c|c|c|c|c|c|c|}
\hline Anatomical region & $L / R$ & $\begin{array}{l}\text { Cluster level P } \\
\text { (corrected) }\end{array}$ & $\begin{array}{l}\text { Size } \\
\text { (voxels) }\end{array}$ & $\begin{array}{l}\text { Voxel level P } \\
\text { (FDR-corrected) }\end{array}$ & $\begin{array}{l}\text { Voxel level P } \\
\text { (uncorrected) }\end{array}$ & \multicolumn{3}{|c|}{$\begin{array}{c}\text { MNI (mm) } \\
\text { x y z }\end{array}$} & $\begin{array}{r}\text { Voxel } \\
\mathrm{Z} \\
\end{array}$ \\
\hline Limbic lobe uncus & Left & 0.015 & 92 & 0.232 & 0.000 & -18 & 2 & -22 & 4.13 \\
\hline Middle Frontal gyrus & Left & 0.083 & 66 & 0.018 & 0.000 & -18 & 46 & -14 & 5.21 \\
\hline Cerebellum posterior & Right & 0.025 & 89 & 0.125 & 0.000 & 26 & -34 & -40 & 3.98 \\
\hline
\end{tabular}

Lobe

Uncorrected $P<0.001,50$ voxels minimum extent. FDR, false discovery rate; MNI, Montreal Neurological Institute. 

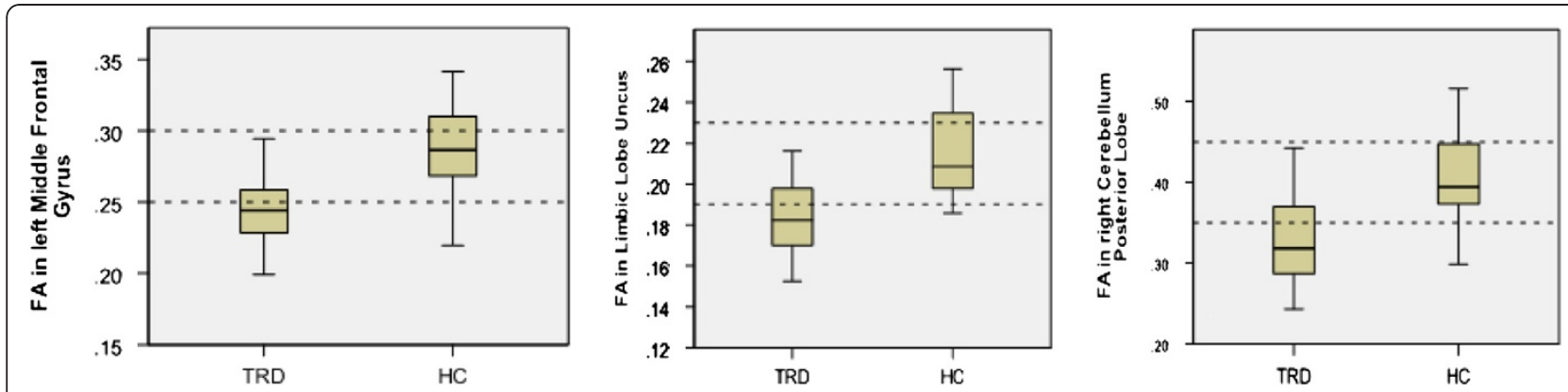

Figure 2 Fractional anisotropy values are significantly decreased at the left middle frontal gyrus, left limbic lobe, and right cerebellum posterior lobe. $P<0.001$. HC, healthy control; TRD, treatment-resistant depression.

neuropsychiatric disorders. A study based on single photon emission computed tomography also suggested a functional impact of cerebellar lesions on cortical functioning through disruption of cerebellar-cerebral connections, indicating a role of the cerebellum in emotional processing [50]. Furthermore, abnormal cerebellar function was reported to be a potential marker of vulnerability to recurrent depression [51]. Based on these studies and our results showing abnormal white matter connections at the left middle frontal gyrus, the left limbic lobe uncus, and the right posterior lobe of the cerebellum, both the cortical-limbic circuit and the cerebellum may contribute to TRD.

Evidence from clinical findings supports the posterior lobe, rather than the anterior lobe, as the cerebellar region of specialization for cognitive and affective processes [52]. With cerebellar damage, there is a tendency toward lateralization in cognitive processing, and right-sided cerebellar lesions often show typical lefthemispheric dysfunctions, including disorders in executive functions, logical reasoning, and language skills [50]. Our results show white matter abnormalities in the left middle frontal gyrus, limbic lobe uncus, and right cerebellum posterior lobe are consistent with the tendency for lateralization [48]. The lateralized cerebral specialization is different between emotional experience and expression, and evidence suggests that positive, approach-related emotions are associated with functions of the left cerebral hemisphere regions, whereas negative, withdrawalrelated emotions are associated with right hemisphere mechanisms [48]. Our results were focused on the left cerebral and right cerebellum posterior lobe, and suggest that TRD may be related to emotional-expression and cognitive-processing disorders.

Numerous studies have demonstrated that the cerebellum is involved in cognitive functions, especially the posterior lobe of the cerebellum, which is considered to be related to executive function, working memory, and language processing $[46,47,50]$. The left middle frontal gyrus is considered an important region for working memory, executive functions, logical reasoning, language skills, and information processing [50,53-55]. In our study, white matter abnormalities were observed simultaneously at the right posterior lobe of the cerebellum and the left middle frontal gyrus, which may enhance the impaired cognitive functions in TRD, and the poorer cognitive functions may be the basis of treatment resistance.

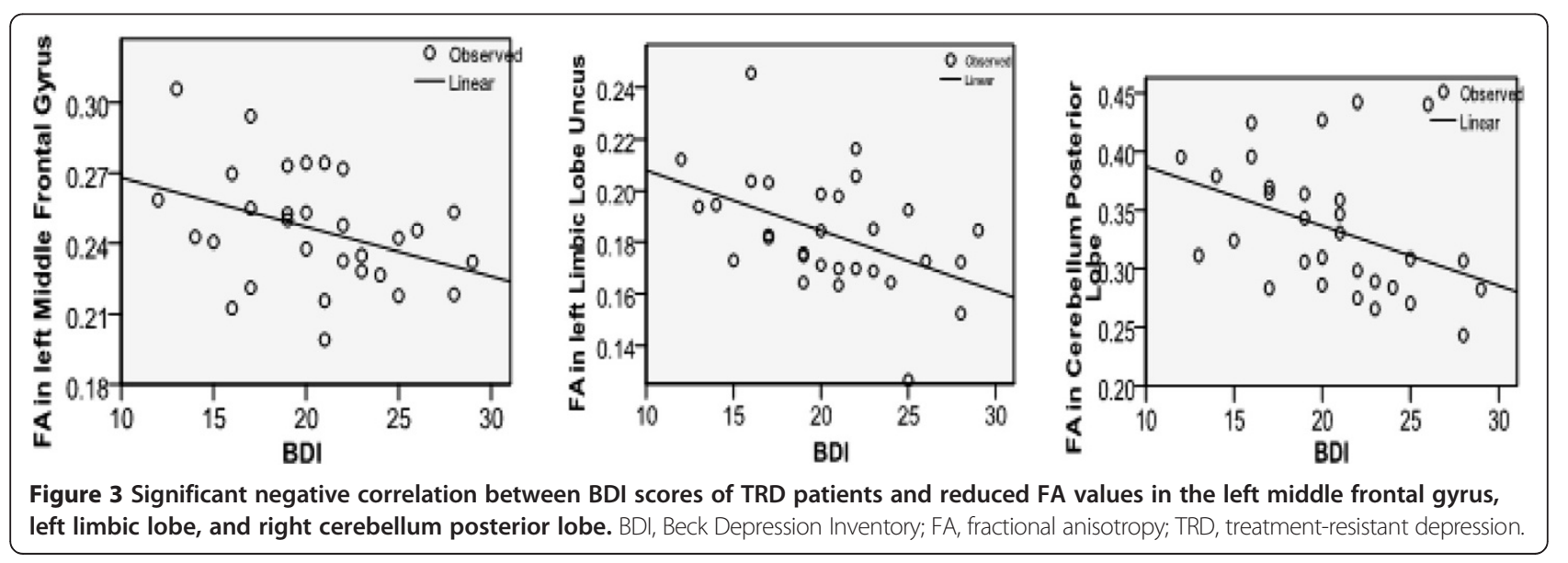


Table 3 Gender differences of mean fractional anisotropy in regions of interest

\begin{tabular}{llllll}
\hline Anatomical region & Gender & $\mathbf{n}$ & Mean & SD & P-value \\
\hline Left limbic lobe uncus & male & 19 & 0.182 & 0.022 & \\
\multirow{3}{*}{ Left middle frontal gyrus } & female & 11 & 0.186 & 0.023 & 0.636 \\
& male & 19 & 0.248 & 0.021 & \\
Right cerebellum posterior lobe & female & 11 & 0.243 & 0.030 & 0.588 \\
& male & 19 & 0.324 & 0.055 & \\
& female & 11 & 0.351 & 0.055 & 0.207 \\
\hline
\end{tabular}

$P>0.05 . \mathrm{SD}$, standard deviation.

In one VBA study of eight refractory depression patients and nine controls, a significant reduction in FA was observed in the frontal lobe, ACC, and temporal lobe in depression patients [22]. However, the sample size of this study was small, and the results did not survive correction, with a threshold set by $P<0.005$ and cluster size $>30$ voxels. In the present study using the same methods, a larger sample size, and a threshold of $P<0.001$ and cluster size $>50$, we found that the cerebellum was also involved in the pathogenesis of TRD. Using the voxel-based method may produce Type I errors, although our results survived a FDR threshold of $P<0.05$ at the cluster level or the voxel level.

We also found a negative correlation between depressive symptom scores and FA values in three areas in TRD patients, further supporting the possibility that damaged white matter integrity was related to disease severity. We did not find a correlation between FA values and age or disease duration, as previously reported [25,27]. These data suggest that reductions of FA in TRD may be related to patient clinical presentation, and less associated with other factors. There is also evidence that gender may influence white matter FA values [56]. However, we found no differences in FA between males to females in TRD patients in the three significant clusters, suggesting that male and female TRD patients exhibit the same pathogenesis.

There are some potential limitations of this study. First, the sample size is small, and these results require replication and further clarification in a larger patient population. Second, new methods such as TBSS should be used in the future to track the precise connections between the cortex, limbic area, and cerebellum, and to examine in more detail the network associated with affection regulation. The VBA as an explorative method is useful for discovering unanticipated or unpredicted neuroanatomical areas, although it can lead to pseudopositive results. Finally, more advanced statistical methods and a more powerful correction should be performed, as our small sample size limited the correction.

\section{Conclusions}

Our DTI results demonstrate, for the first time, a role of the cerebellum in the pathogenesis of TRD. We suggest that the changes in white matter FA in TRD patients are similar to MDD, implicating abnormalities of the cortical-limbic circuits, but are also associated with the cerebellum. The pathogenesis of TRD may be related to abnormalities of cortical-limbic-cerebellar white matter networks. Future studies with larger sample sizes and better methods such as TBSS are required to replicate these results.

\section{Competing interests}

The authors declare that they have no competing interests.

\section{Authors' contributions}

Authors HP and $\mathrm{HZ}$ designed the study and developed the protocols. $\mathrm{LL}$ and YN are tutors of HP. Authors HP, HZ, YZ, LZ, and HY carried out literature searches and analyses. Authors BS, JL, JZ, ZL, and ZZ performed statistical analyses and prepared the first draft of the manuscript. All authors read and approved the final manuscript.

\section{Acknowledgements}

We thank all the patients and control subjects who took part in our studies. This study was supported by grants from the National Natural Science Foundation of China (30830046 \& 81171286 to Lingjiang Li, C090104 to Yuping Ning), the National Science and Technology Program of China (2007BAl17B02 to Lingjiang Li), the National 973 Program of China (2009CB918303 to Lingjiang Li, and 2007CB512308 to Zhijun Zhang), the Chinese Ministry of Education (20090162110011 to Lingjiang Li), the Medical Research Foundation of Guangdong, China (A2012523 to Hongjun Peng), the Science and Technology Program of Guangzhou, China (2010Y1-C631 to Yuping Ning), the Science and Technology Program of Guangdong "China (2012B031800015 to Hongjun Peng), and the National Hi-Tech Research and Development Program of China (863 program: 2008AA02Z413 to Zhijun Zhang).

\section{Author details}

${ }^{1}$ Mental Health Institute, The 2nd Xiangya Hospital, Central South University, No. 139 Renmin Zhong Road, Changsha 410011, China. ${ }^{2}$ Guangzhou Psychiatric Hospital, Affiliated Hospital of Guangzhou Medical College, Guangzhou, China. ${ }^{3}$ Guangdong Mental Health Institute, Guangdong General Hospital, Guangzhou, China. ${ }^{4}$ Key Laboratory of Nuclear Analysis, Institute of High Energy Physics, Chinese Academy of Sciences, Beijing, People's Republic of China. ${ }^{5}$ Department of Radiology, The Second Xiangya Hospital of Central South University, Changsha, Hunan, China. ${ }^{6}$ The Department of Neuropsychiatry and Institute of Neuropsychiatric Research, Affiliated ZhongDa Hospital of Southeast University, Nanjing, China. ${ }^{7}$ Chinese University of Hong Kong, Hong Kong, China.

Received: 30 August 2012 Accepted: 21 February 2013

Published: 2 March 2013

\section{References}

1. Moussavi S, Chatterji S, Verdes E, Tandon A, Patel V, Ustun B: Depression, chronic diseases, and decrements in health: results from the World Health Surveys. Lancet 2007, 370(9590):851-858.

2. Amital D, Fostick L, Silberman A, Beckman M, Spivak B: Serious life events among resistant and non-resistant MDD patients. J Affect Disord 2008, 110(3):260-264.

3. Ananth J: Treatment-resistant depression. Psychother Psychosom 1998, 67(2):61-70.

4. Fava M, Davidson KG: Definition and epidemiology of treatment-resistant depression. Psychiatr Clin N Am 1996, 19(2):179-200.

5. Keller MB: Issues in treatment-resistant depression. Journal of Clinical Psychiatry 2005, 66:5-12.

6. Rush AJ, Thase ME, Dube S: Research issues in the study of difficult-to -treat depression. Biol Psychiatry 2003, 53(8):743-753. 
7. Zhang TJ, Wu QZ, Huang XQ, Sun XL, Zou K, Lui S, Liu F, Hu JM, Kuang WH, Li DM, et al: Magnetization transfer imaging reveals the brain deficit in patients with treatment-refractory depression. J Affect Disord 2009, 117(3):157-161.

8. Drevets $W C$, Price $J$, Furey ML: Brain structural and functional abnormalities in mood disorders: implications for neurocircuitry models of depression. Brain Structure \& Function 2008, 213(1-2):93-118.

9. Lim KO, Helpen JA: Neuropsychiatric applications of DTI - a review. NMR Biomed 2002, 15(7-8):587-593.

10. Taylor WD, Hsu E, Krishnan KRR, MacFall JR: Diffusion tensor imaging: Background, potential, and utility in psychiatric research. Biol Psychiatry 2004, 55(3):201-207.

11. Fields RD: White matter in learning, cognition and psychiatric disorders. Trends Neurosci 2008, 31(7):361-370.

12. Sexton CE, Mackay CE, Ebmeier KP: A Systematic Review of Diffusion Tensor Imaging Studies in Affective Disorders. Biol Psychiatry 2009, 66(9):814-823.

13. Beyer JL, Taylor WD, MacFall JR, Kuchibhatla M, Payne ME, Provenzale JM, Cassidy F, Krishnan KRR: Cortical white matter microstructural abnormalities in bipolar disorder. Neuropsychopharmacology 2005, 30(12):2225-2229.

14. Cullen KR, Klimes-Dougan B, Muetzel R, Mueller BA, Camchong J, Houri A, Kurma S, Lim KO: Altered White Matter Microstructure in Adolescents With Major Depression: A Preliminary Study. J Am Acad Child Adolesc Psychiatry 2009, 49(2):173-183.

15. Li LJ, Ma N, Li ZX, Tan LW, Liu J, Gong GL, Shu N, He Z, Jiang TZ, Xu L: Prefrontal white matter abnormalities in young adult with major depressive disorder: A diffusion tensor imaging study. Brain Research 2007, 1168:124-128.

16. Yang $Q$, Huang XB, Hong N, Yu X: White matter microstructural abnormalities in late-life depression. Int Psychogeriatr 2007, 19(4):757-766.

17. $X I$ L, Wang YZ, Liu HH, Liu ZN, Zhou WB: Diffusion tensor imaging and resting state functional magnetic resonance imaging on young patients with major depressive disorder. Zhong Nan Da Xue Xue Bao Yi Xue Ban 2010, 35(1):25-31.

18. Fagiolini A, Kupfer DJ: Is treatment-resistant depression a unique subtype of depression? Biol Psychiatry 2003, 53(8):640-648.

19. Phillips ML, Drevets WC, Rauch SL, Lane R: Neurobiology of emotion perception II: Implications for major psychiatric disorders. Biol Psychiatry 2003, 54(5):515-528.

20. Nauta WJH, Domesick VB: Afferent and efferent relationships of the basal ganglia. Ciba Foundation Symposia 1984, 107:3-29.

21. Ongur D, Ferry AT, Price JL: Architectonic subdivision of the human orbital and medial prefrontal cortex. J Comp Neurol 2003, 460(3):425-449.

22. Li CX, Sun XL, Zou K, Yang H, Huang XQ, Wang YQ, Liu S, Li DM, Zhou L, Chen HF: Voxel based Analysis of DTI in Depression Patients. Inter J Magn Reson Imaging 2007, 01:043-048.

23. Zheng HR, Zhang L, Li LJ, Liu P, Gao JL, Liu XY, Zou JA, Zhang Y, Liu J, Zhang ZJ, et al: High-frequency rTMS treatment increases left prefrontal myo-inositol in young patients with treatment-resistant depression. Prog Neuropsychopharmacol Biol Psychiatry 2010, 34(7):1189-1195.

24. Beck AT, Beamesderfer A: Assessment of depression: the depression inventory. Mod Probl Pharmacopsychiatry 1974, 7:151-169.

25. Liao Y, Tang J, Ma M, Wu Z, Yang M, Wang X, Liu T, Chen X, Fletcher PC, Hao W: Frontal white matter abnormalities following chronic ketamine use: a diffusion tensor imaging study. Brain 2010, 133:2115-2122.

26. Peng $H$, Zheng H, Li L, Liu J, Zhang Y, Shan B, Zhang L, Yin Y, Li W: Highfrequency rTMS treatment increases white matter FA in the left middle frontal gyrus in young patients with treatment-resistant depression. J Affect Disord 2012, 136:249-257.

27. Tang J, Liao Y, Zhou B, Tan C, Liu T, Hao W, Hu D, Chen X: Abnormal anterior cingulum integrity in first episode, early-onset schizophrenia: a diffusion tensor imaging study. Brain research 2010, 1343:199-205.

28. Genovese CR, Lazar NA, Nichols T: Thresholding of statistical maps in functional neuroimaging using the false discovery rate. Neurolmage 2002, 15(4):870-878

29. Hao Y, Yan Q, Liu H, Xu L, Xue Z, Song X, Kaneko Y, Jiang T, Liu Z, Shan B: Schizophrenia patients and their healthy siblings share disruption of white matter integrity in the left prefrontal cortex and the hippocampus but not the anterior cingulate cortex. Schizophr Res 2009,

114(1-3):128-135.
30. Abe O, Yamasue H, Kasai K, Yamada H, Aoki S, Inoue H, Takei K, Suga M, Matsuo K, Kato T, et al: Voxel-based analyses of gray/white matter volume and diffusion tensor data in major depression. Psychiatry ResearchNeuroimaging 2009, 181(1):64-70.

31. Kempton MJ, Salvador Z, Munafo MR, Geddes JR, Simmons A, Frangou S, Williams SCR: Structural neuroimaging studies in major depressive disorder: meta-analysis and comparison with bipolar disorder. Arch Gen Psychiatry 2011, 68:675.

32. Kieseppa T, Eerola M, Mantyla R, Neuvonen T, Poutanen VP, Luoma K, Tuulio-Henriksson A, Jylha P, Mantere O, Melartin T, et al: Major depressive disorder and white matter abnormalities: A diffusion tensor imaging study with tract-based spatial statistics. J Affect Disord 2009, 120(1-3):240-244

33. Konarski JZ, Mclntyre RS, Kennedy SH, Soczynska JK, Ketter TA: Volumetric neuroimaging investigations in mood disorders: bipolar disorder versus major depressive disorder. Bipolar disorders 2008, 10:1-37.

34. Versace A, Almeida JRC, Quevedo K, Thompson WK, Terwilliger RA, Hassel S, Kupfer DJ, Phillips ML: Right Orbitofrontal Corticolimbic and Left Corticocortical White Matter Connectivity Differentiate Bipolar and Unipolar Depression. Biol Psychiatry 2010, 68(6):560-567.

35. Zhu XL, Wang XA, Xiao J, Zhong MT, Liao JA, Yao SQ: Altered white matter integrity in first-episode, treatment-naive young adults with major depressive disorder: A tract-based spatial statistics study. Brain Research 2011, 1369:223-229

36. Mayberg HS: Modulating dysfunctional limbic-cortical circuits in depression: towards development of brain-based algorithms for diagnosis and optimised treatment. Br Med Bull 2003, 65:193-195.

37. Mayberg HS: Limbic-cortical dysregulation: A proposed model of depression. J Neuropsychiatry Clin Neurosci 1997, 9(3):471-481.

38. Mayberg HS: Modulating limbic-cortical circuits in depression: targets of antidepressant treatments. Semin Clin Neuropsychiatry 2002, 7(4):255-256.

39. Tekin S, Cummings JL: Frontal-subcortical neuronal circuits and clinical neuropsychiatry - An update. J Psychosom Res 2002, 53:647-654.

40. Dietrich DE, Bonnemann C, Emrich HM: Cortico-limbic mechanisms of affect regulation in the therapy of depression. Psychiatr Prax 2007, 34:S287-S291.

41. Goldapple K, Segal Z, Garson C, Lau M, Bieling P, Kennedy S, Mayberg H: Modulation of cortical-limbic pathways in major depression - Treatmentspecific effects of cognitive behavior therapy. Arch Gen Psychiatry 2004, 61(1):34-41

42. Grace AA: Disruption of cortical-limbic interaction as a substrate for comorbidity. Neurotox Res 2006, 10(2):93-101.

43. Wager TD, Davidson ML, Hughes BL, Lindquist MA, Ochsner KN: Prefrontalsubcortical pathways mediating successful emotion regulation. Neuron 2008, 59(6):1037-1050.

44. Seminowicz D, Mayberg H, McIntosh A, Goldapple K, Kennedy S, Segal Z, Rafi-Tari S: Limbic-frontal circuitry in major depression: a path modeling metanalysis. Neurolmage 2004, 22:409-418.

45. Mervaala E, Fohr J, Kononen M, Valkonen-Korhonen M, Vainio P, Partanen K, Partanen J, Tiihonen J, Viinamaki H, Karjalainen AK, et al: Quantitative MRI of the hippocampus and amygdala in severe depression. Psychol Med 2000, 30(1):117-125.

46. Glickstein M, Doron K: Cerebellum: Connections and Functions. Cerebellum 2008, 7(4):589-594.

47. Schmahmann JD: The Role of the Cerebellum in Cognition and Emotion: Personal Reflections Since 1982 on the Dysmetria of Thought Hypothesis, and Its Historical Evolution from Theory to Therapy. Neuropsychol Rev 2010, 20(3):236-260.

48. Lee GP, Meador KJ, Loring DW, Allison JD, Brown WS, Paul LK, Pillai JJ, Lavin TB: Neural substrates of emotion as revealed by functional magnetic resonance imaging. Cogn Behav Neurol 2004, 17(1):9-17.

49. Doron KW, Funk CM, Glickstein M: Fronto-cerebellar circuits and eye movement control: A diffusion imaging tractography study of human cortico-pontine projections. Brain Research 2009, 1307:63-71.

50. Baillieux H, De Smet HJ, Dobbeleir A, Paquier PF, De Deyn PP, Marien P: Cognitive and affective disturbances following focal cerebellar damage in adults: A neuropsychological and SPECT study. Cortex 2009, 46(7):869-879.

51. Katharine A, Smith AP, Cowen PJ, Mccleery JM, Goodwin GM, Smith S, Tracey I, Matthews PM: Cerebellar responses during anticipation of noxious stimuli in subjects recovered from depression: functional magnetic resonance imaging study. Br J Psychiatry 2002, 181:411-415. 
52. Stoodley CJ, Schmahmann JD: Evidence for topographic organization in the cerebellum of motor control versus cognitive and affective processing. Cortex 2009, 46(7):831-844.

53. Belger A, Puce A, Krystal JH, Gore JC, Goldman-Rakic P, McCarthy G: Dissociation of mnemonic and perceptual processes during spatial and nonspatial working memory using fMRl. Human Brain Mapping 1998, 6(1):14-32.

54. Liu CL, Hue CW, Chen CC, Chuang KH, Liang KC, Wang YH, Wu CW, Chen $\mathrm{JH}$ : Dissociated roles of the middle frontal gyri in the processing of Chinese characters. Neuroreport 2006, 17(13):1397-1401.

55. Miller EK, Cohen JD: An integrative theory of prefrontal cortex function. Annu Rev Neurosci 2001, 24:167-202.

56. Yao ZJ, Liu HY, Teng GJ, Lu Q, Wang L, Cao YX: The preliminary study of gender difference in brain white matter in major depression. Chin J Nerv Ment Dis 2008, 34:161-164.

doi:10.1186/1471-244X-13-72

Cite this article as: Peng et al:: Abnormalities of cortical-limbic-cerebellar white matter networks may contribute to treatment-resistant

depression: a diffusion tensor imaging study. BMC Psychiatry 2013 13:72.

\section{Submit your next manuscript to BioMed Central and take full advantage of:}

- Convenient online submission

- Thorough peer review

- No space constraints or color figure charges

- Immediate publication on acceptance

- Inclusion in PubMed, CAS, Scopus and Google Scholar

- Research which is freely available for redistribution 